\title{
Application of Cellular Automata and Lattice Boltzmann Methods for modelling of Additive Layer Manufacturing
}

\author{
D. Svyetlichnyy ${ }^{\text {a*, M. Krzyzanowski }}{ }^{\mathrm{a}, \mathrm{b}}$, R. Straka ${ }^{\mathrm{a}}$, L. Lach $^{\mathrm{a}}$ and W.M. Rainforth \\ ${ }^{a}$ AGH University of Science and Technology, Faculty of Metals Engineering and Industrial \\ Computer Science, al. Mickiewicza 30, Krakow, Poland \\ ${ }^{b}$ Birmingham City University, Faculty Computing, Engineering \& the Built Environment, Millenium \\ Point, Curzon Street, Birmingham B4 7XG, UK \\ c The University of Sheffield, Department of Materials Science and Engineering, Mappin Street,
} Sheffield S1 3JD, UK

\begin{abstract}
Purpose - The holistic numerical model based on cellular automata (CA) and Lattice Boltzmann methods (LBM) is being developed as part of an integrated modelling approach applied to study the interaction of different physical mechanisms in laser assisted additive layer manufacturing (ALM) of orthopaedic implants. Several physical events occuring in sequence or simultaneously are considered in the holistic model. They include a powder bed deposition, laser energy absorption and heating of the powder bed by the moving laser beam leading to powder melting or sintering, fluid flow in the melted pool, flow through partly or not melted material and solidification.

Design/methodology/approach - The mentioned physical events are accompanied by heat transfer in solid and liquid phases including interface heat transfer at the boundaries. The sintering/melting model is being developed using LBM as an independent numerical method for hydrodynamic simulations originated from lattice-gas cellular automata (LGCA). It is going to be coupled with the CA based model of powder bed generation.

Findings - The entire laser assisted ALM process has been analised and divided on several stages considering the relevant physical phenomema. The entire holistic model consisting of four interrelated submodels has currently been developed to a different extent. The submodels include the CA based

\footnotetext{
* Corresponding author.

E-mail address: svetlich@metal.agh.edu.pl (Dmytro Svyetlichnyy)
} 
model of powder bed generation, the LBM-CA based model of heat exchange and transfer, the thermal solid-liquid interface model and the mechanical solid-liquid interface model for continuous liquid flow.

Practical implications - The results obtained can be used to explain the interaction of the different physical mechanisms in ALM, which is intensively developing field of advanced manufacturing of metal, non-metal and composite structural parts for instance in bio-engineering among others. The proposed holistic model is considered to be a part of the integrated modelling approach being developed as a numerical tool for investigation of the co-operative relashionships between multiphysical phenomena occurred in sequence or simultaneousely during heating of the powder bed by the moving high energy heat source leading to selective powder sintering or melting, fluid flow in the melted pool and through partly (or not) melted material and also to solidification. The model is compatible with the earlier developed CA based model for generation of the powder bed allowing for decrease of the numerical noise.

Originality/value - The present results are original and new for the study of the complex relathionships between multifysical phenomena occurring during ALM process based on selective laser sintering or melting (SLS/SLM) including fluid flow and heat transfer among others identified as crucial for obtaining the desirable properties.

Keywords - Additive Layer Manufacturing, Lattice Boltzmann Method, Cellular Automata, Selective Laser Sintering, Selective Laser Melting, Modelling.

Paper type - Research paper

\section{Nomenclature}

\section{Roman letters}

$\begin{array}{ll}c & =\text { specific heat } \\ \boldsymbol{e} & =\text { phase space variable } \\ \left\{\mathbf{e}_{i}, i=1, \ldots, \mathrm{b}\right\} & =\text { set of discrete velocities } \\ \boldsymbol{F} & =\text { external force } \\ f(\boldsymbol{x}, \boldsymbol{v}, t) & =\text { particles distribution function } \\ f_{i}^{e q}(\mathbf{x}, t) \equiv f_{i}^{e q}(\rho, \mathbf{v}) & =\text { equilibrium distribution function of } i^{\text {th }} \text { discrete velocity } \\ g_{i} & =\text { distribution function for the thermal (diffusion-convection) problem } \\ k & =\text { thermal conductivity } \\ m & =\text { mass }\end{array}$




\section{Greek symbols}

$\rho$

$\rho v$

$\tau$

$\tau_{T}$

$\Omega$

Subscripts

$i$

T

Superscripts

$e q$

\section{Introduction}

ALM is an intensively developing field of research for the manufacture of bioresorbable scaffolds in tissue engineering for repair of skeletal defects, among many others applications (Eshraghi and Das, 2012). ALM methods enable effective control over the structure of the scaffold, which in turn dictates the geometry of the newly formed tissue. Implants made by ALM methods can have increased longevity by providing more secure fixation using tailored porosity, which cannot be achieved by conventional manufacturing techniques (Mercuri et al., 1995). The paper deals with development of the holistic numerical model based on cellular automata (CA) and Lattice Boltzmann methods (LBM) for simulation of selective laser sintering/melting (SLS/SLM) as a part of ALM technology. The approach is considered to be useful for process development, especially for qualification of new materials and investigation of the different technological aspects. The modern human implants consist of materials having different mechanical and thermal properties. They are manufactured in several stages. The material melted at the highest temperature is formed firstly following by materials with 
lower temperature. However, to meet a complicated shape of the final products and often the stringent property requirements (for example, porosity), the one process stage often is required. Development of such one-stage processes is a significunt technological and scientific challenge. Undoubtedly, a detailed theoretical analysis based on mathematical modelling of the entire manufacturing process is a crucial stage in the process development. A typical SLS/SLM process consists of four basic stages, which take place simultaneously or sequentially, namely a powder bed deposition stage; a heating and melting by laser beam stage; a flow of molten material between unmelted and semi-melted particles of the bed of powder and across the solid basement; and a solidification stage. The sub-models relevant to the mentioned four stages are being developed and described in the paper. The last three processes are accompanied by heat transfer between solid, liquid and gas phases including convection and thermal radiation. The four principal tasks are considered and numerically solved in the modelling, such as heat transfer from laser beam to opaque, transparent and translucent material; material melting; flow of the molten material and solidification. The sintering/melting model is developed based on cellular automata (CA) and Lattice Boltzmann methodology (LBM). The holistic approach presented in the paper is a numerical model that addresses all the above-mentioned issues for simulation and modelling of the laser assisted ALM process. In the 1980s, new ALM technologies are appeared along with conventional subtractive methods. Many of them are based on selective laser sintering/melting (SLS/SLM). They used a moving laser beam to selectively sinter (or melt) the deposited polymer and/or metal composite powder materials into a three-dimensional part of the final product. The process is schematically shown in Fig.1. The product is manufactured in the chamber located on a movable platform that adjusts its height equal to the thickness of the forming layer. Powder is added from the the powder feed device and levelled with a roller or using other methods. Powder is deposited on the top of the powder bed in the build chamber, which contains the previously sintered (or melted and solidified) layer of the product and an unprocessed amount of powder. Then, a new layer is melted or sintered with the laser beam and the cycles are repeated untill the final product is obtained.

Different ALM methods have been used to prepare scaffolds, including SLS/SLM, Fused Deposition Modelling (FDM) and Precision Extrusion Deposition (PED) presented by Domingos et al. (2012), Krzyzanowski et al. (2016) and Lam et al. (2007). ALM methods enable effective control over the structure of the scaffold, which in turn dictates the geometry of the newly formed tissue. Moreover, the ability of SLS/SLM to manufacture anatomically shaped scaffolds with designed microstructure made of bioactive and bioresorbable composite materials at high filler loadings allows for fabrication of scaffolds with a high degree of geometric complexity and enables the direct conversion of the digital representation of any object into its physical realization. The method also enables the development of patient and tissue specific reconstruction strategies (Williams et al., 2005 and Wu et al., 2008). SLS has the potential to construct bioactive implants due to its capability of processing polymers, ceramics, metals and their composites. Bioresorbable scaffolds with mechanical properties suitable for bone tissue engineering have been fabricated from polycaprolactone (PCL) 
and hydroxyapatite (HA) by SLS. HA is a calcium phosphate (CaP) material that is chemically similar to the inorganic component of bone matrix and can promote apatite deposition and bone cell attachment (Hench and Polak, 2002).

The aim of this work is to develop a structure of the holistic numerical model based on cellular automata (CA) and Lattice Boltzmann methods (LBM) applicabale for studying the interaction of the different physical mechanisms in ALM of orthopaedic implants. The model is considered to be a part of the integrated modelling approach being developed for investigation of the co-operative relashionships between multiphysical phenomena occurred in sequence or simultaneousely during heating of the powder bed by the moving high energy heat source leading to selective powder sintering or melting, fluid flow in the melted pool and through partly (or not) melted material and also to solidification. The model should also be compatible with the earlier developed CA based model for generation of the powder bed.

\section{Development of homogenous holistic model for ALM}

The process described above can be simplified for simulation purpose, as it is schematically shown in Fig. 2. It can be described as a sequence of the following stages, namely: powder deposition, pause, laser treatment and pause. The sequence is repeated. The following five basic processes or phenomena are considered and modelled:

- $\quad$ powder bed generation (PBG),

- $\quad$ laser beam treatment (LBT),

- $\quad$ melting $(\mathrm{M})$,

- free flow of melted material (LF),

- $\quad$ solidification $(\mathrm{S})$.

The mentioned five processes take place sequentially or simultaneously. These processes and phenomena can be simulated and modelled using four of the following models (Fig. 2):

1. PBG model,

2. Heat exchange and transfer model (HE\&T),

3. Model of thermal solid-liquid interface (SLIT),

4. Model of mechanical solid-liquid interface (SLIM).

\section{Homogenous and multi-scale models}

Currently, large holistic models use multi-scale approaches that rely on a combination of several models operating on different principles and (or) in different scales. For example, different models based on either CA or discrete element method (DEM) can be used for simulation of the powder bed generation. Thermal processes can be modelled using finite element (FE), finite difference (FD) or Lattice Boltzmann methods (LBM). While DEM or LBM can be used to model liquid flows, and FEM or CA for melting and solidification. 
There are many different variants can be used to create holistic model, for example, DEM-FEM-CA based model. Most of the combinations are significantly inhomogeneous or heterogeneous multi-scale models that would require complicated interfaces between the various components. It almost eliminates the possibility to complete full scale calculations within a single numerical model without running independently the individual modules and combining them afterwards. As a result, difficulties and limitations for development of such multiscale heterogeneous holistic models arise and, at the same time, arises the question of feasibility of such approaches. However, development of the holistic model based entirely on one or two homogeneous methods allows for modelling very complex physical phenomena accompanying the manufacturing process and for elimination of the complicated interface. The approaches based on CA and Lattice Boltzmann methods (CA-LBM) are among the methods that satisfy the above mentioned criteria.

The model of the material flow is the most important and crucial model from the point of view of the structure homogeneity of the holistic model. The solution of the Navier-Stokes equations, which describe the principle of conservation of momentum for moving fluid, is often associated with a variety of problems using FE, FD and other approaches. In particular, the important problem is to obtain stability of the solutions in modelling of turbulent flow or advection without diffusion. In contrast, the LBM is free of this weakness and considered to be the most effective for modelling flow of liquid, gas, mixtures and suspensions. Kuwata and Suga (2016) discussed direct numerical simulation carried out using LBM for modelling of the turbulent flow over a permeable porouse layer. It also allows for modelling of heat transfer, heat exchange and diffusion that take place simultaneously with the movement of the liquid (gas). In the recent work of Eshghinejadfard and Thévenin (2016), the LBM is applied to investigate heat transfer phenomena in particulate flows. With this aim in mind, the direct-forcing and direct-heating immersed boundary (IB) method have been applied for calculations of the hydrodynamic force and energy exchange between the particles and the fluid. Except for powder deposition and partly melting-solidification, all other processes and phenomena occurring during the SLS/SLM can be modelled using LBM. While CA seems to be the only method that allows for modelling of these exepted cases, such as powder deposition, etc and it does not affect the homogeneity of the holistic model. The model based on CA and LBM does not require a complicated interface to connect the various models of different processes and phenomena.

\section{CA-LBM based model}

The model operates in a common domain. Number of processes and phenomena that can be modelled simultaneously and also accuracy of the simulation is defined with a number of variables associated with the point, cell or node in the same domain. Implementation of a new process or phenomenon into such model leads 
to addition of the new variables and appropriate algorithms without complicated communication with different parts of the model, as it takes place in multiscale models. Because LBM is originated from the CA, a combination of these two methods into one single holistic model is easy, simple and straightforward; and it seems to be optimal. Therefore, it has been decided that the holistic model for simulation of the entire technological process is going to be based on CA and LBM. The approach will simplify communication between separate models of the particular processes and phenomena taking place during the laser assisted ALM.

$\mathrm{CA}$ is a well-known universal numerical method for modelling and simulation of the different processes and phenomena, presented as development of complex dynamic system with definite states of their elements and possibility of discretization of space and time. The method is based on informational interaction of the universal automata both between themselves and with a defined environment (neighborhood). The modified algorithm of CA, known as Frontal Cellular Automata (FCA) proposed by Svyetlichnyy (2013) and described in detail elsewhere (Svyetlichnyy $\left.{ }^{\mathrm{a}}, 2014\right)$, allows for significant acceleration of the calculations. CA and FCA have been applied for modelling of microstructure evolution, for example for creation of initial microstructure $\left(\right.$ Svyetlichnyy $^{\mathrm{b}}$, 2014), crystallization (Svyetlichnyy, 2013), phase transformation (Svyetlichnyy $^{\mathrm{a}}$, 2014) and grain refinement (Svyetlichnyy, 2008 and 2010). Also, several FCA-based application have been developed such as solidification in the continuous casting, the hot flat and shape rolling, the roll-bonding process and the multiaxial compression (Svyetlichnyy, 2015). CA has also been proved to be an effective tool for simulation of powder bed generation (Krzyzanowski et al., 2016).

Originated from the CA application known as lattice gas automata (LGA), LBM is considered to be a simplified fictitious molecular dynamics model in which space, time, and particle velocities are all discrete. It solves the statistical Boltzmann equation. High memory requirements, lack of effective algorithms, limitation of parallel computations and other reasons significantly impeded development of both LBM and CA for many years. The following three classes of thermal LBM have been proposed to fluid flow with heat transfer, namely: the multi-speed, the passive scalar and the double distribution function approach. For instance, the details of the recently developed stable and accurate finite volume formulation of the thermal LBM are presented by Zarghami et el. (2014). Parallel programming environment using graphics cards - CUDA (Compute Unified Device Architecture), developed by NVIDIA in 2007, is almost ideal for LBM applications due to its specific algorithmic structure. This development has entirely changed the status of LBM, from being little useful to a highly efficient and very effective method; and accelerated its development, expanding the area of the applications. Tremendous increase of interest in this methodology is anticipated in the coming years.

\section{Powder bed generation model}


Multi material laser assisted ALM processes deal with different powder materials characterized by particle shape (morphology), size, and distribution (granulometry). The overall particle shape can be classified as atomized, spherical or spheroidal; and granular, or flake. Most of the metal powders used in ALM can be considered as spherical for modelling purposes, and the structure of the powder bed for such materials can be well predicted using the recently developed CA based model described in details elsewhere (Krzyzzanowski et $a l .$, 2016). The model has been verified comparing the simulation results with the experimental data derived from studying of Ti-6Al-4V powder disposition into a cylinder. The simulated particles were almost spherical bodies. The coordinates of the center and diameter were determined using XCT (X-ray Computer Tomography) analysis (Tammas-Williams et al., 2015). The particles having the same shape and size were mixed and deposited with random coordinate into the cylinder of the same diameter during the simulation. The average simulated deposit density was about $3.2 \%$ higher than the experimentally obtained packing density, which can be considered as a good agreement.

However, not all materials used for ALM have spherical particle shapes, e.g. polymers. Currently, the various shapes of both rigid and deformable particles, a typical for bioactive glass powder were implemented into the CA code. The particle deposition model has been extended to generation of the arbitrary particles shape and more flexible control of the particle characteristics and distribution functions. As a result, the model of powder bed generation allows for deposition of the irregular shape particles with the required packing density (Fig. 3).

\section{Heat exchange and transfer model}

The next model, which is under development, is a model of heat transfer from the laser beam to opaque, transparent and translucent material and its adaptation to CA-LBM model. Here, the approach widely used in the finite element method (FEM) and finite discrete method (FDM) is adopted to the CA-LBM-based model. In the case of opaque materials, appropriate boundary conditions are applied on the surface of the particles. In other cases, internal volume heat source taking into account the intensity of the absorbed light energy is introduced. There are three main approaches to the thermal LBM (TLBM): the multi-speed method (extended velocity set LBM), double population methods using two populations of distribution functions to describe fluid flow and heat transfer or hybrid methods.

\section{Thermal solid-liquid interface}

FEM, FDM, volume element method (VEM), CA and LBM are used for modelling of material melting and solidification. In most of these approaches, the important problem is consideration of transformation heat at a melting-solidification point. For this purpose, the effective heat capacity method is widely used. The method is efficient when difference between the solidus and liquidus temperature is 
significant. On the other hand, the smaller the difference, the less effecient it becomes. The method does not work when the liquidus and solidus are the same temperature. There is another known method taking into account transformation heat by introducing a temporary heat source. Here, the stability problems may arise, which are usually solved with reduction of the time step, when heat is distributed along the time of several time steps. Although, such approach can be justified for phase transformations in solid state characterized by significant overheating or overcooling, it doesn't appropriate for modelling of the typical changes of aggregation state, such as melting and solidification of the most materials. In contrast, the transformation heat can be directly taken into account in CA by introducing additional states and variables (Mikhaylovska, 2008). Thus, the effects associated with computational, numerical and algorithmic errors do not arise. The CA algorithm can be easily implemented into LBM. In this case, melting is modelled as a result of direct heating by a laser beam, or by transfering the heat from the molten material. In the second case, the heat transfer equation is applied. The same CA-LBM based model is used both for modelling of the material solidification and melting. Thus, the balance of heat flow at the solid-liquid interface decides on the direction of interface movement, i.e. melting or solidification takes place.

The focal point of the LBM is a numerical solution of the Boltzmann transport equation:

$$
\frac{\partial f}{\partial t}+\frac{\partial f}{\partial \mathbf{x}} \cdot \mathbf{e}+\frac{\mathbf{F}}{m} \cdot \frac{\partial f}{\partial \mathbf{e}}=\Omega
$$

where $f(\boldsymbol{x}, \boldsymbol{v}, t)$ is the particles distribution function, $\boldsymbol{x}$ and $\boldsymbol{e}$ are the phase space variables, $t$ is the time, $\boldsymbol{F}$ is the external force (e.g., gravity), $m$ is mass and $v$ is a vector of the macroscopic velocity. Using the ChapmanEnskog or Grad expansions (Kremer, 2010), it can be proved that the equation is consistent with the NavierStokes equations in the hydrodynamic limit. Particle velocity space can be reduced to a set of discrete velocities $\left\{\mathbf{e}_{i}, i=1, \ldots, \mathrm{b}\right\}$ and the construction of the lattice, on which the calculations are carried out. Usually the equation is approximated along characteristic velocity vectors using trapezoidal rule in space and explicit Euler scheme for the time derivative:

$$
f_{i}\left(\mathbf{x}+\mathbf{e}_{i}, t+1\right)=f_{i}(\mathbf{x}, t)-\frac{1}{\tau}\left[f_{i}(\mathbf{x}, t)-f_{i}^{e q}(\mathbf{x}, t)\right]+F_{i},
$$

where $f_{i}^{e q}(\mathbf{x}, t) \equiv f_{i}^{e q}(\rho, \mathbf{v})$ is the equilibrium distribution function of $i^{\text {th }}$ discrete velocity and $\tau$ is the relaxation time. Eq. (2) is solved numerically in two consequtive stages, namely: streaming

$$
f_{i}^{\text {in }}(\mathbf{x}, t)=f_{i}^{\text {out }}\left(\mathbf{x}-\mathbf{e}_{i}, t-1\right),
$$

and collision:

$$
f_{i}^{\text {out }}(\mathbf{x}, t)=f_{i}^{\text {in }}(\mathbf{x}, t)-\frac{1}{\tau}\left[f_{i}^{\text {in }}(\mathbf{x}, t)-f_{i}^{e q}(\mathbf{x}, t)\right]+F_{i}
$$

During the streaming stage, expressed by eq. (3), the distribution functions are transferred to their neighbour lattice site according to their velocity vectors and approach their equilibrium distributions in the collision step expressed by eq (4). The incoming and outgoing distribution functions, i.e. the functions before and after 
collision, are denoted correspondingly with "in" and "out" indices. The macroscopic density $\rho$ and momentum $\rho v$ in a cell are the $0^{\text {th }}$ and $1^{s t}$ moments of the distribution functions:

$$
\rho=\sum_{i=0}^{b} f_{i}, \quad \rho \mathbf{v}=\sum_{i=0}^{b} f_{i} e_{i}
$$

The viscosity $v$ is defined by the following equation:

$$
v=\frac{1}{3}\left(\tau-\frac{1}{2}\right)
$$

The distribution function $g_{i}$ for the thermal (diffusion-convection) problem can be expressed in the following form:

$$
g_{i}\left(\mathbf{x}+\mathbf{e}_{i}, t\right)=g_{i}(\mathbf{x}, t)-\frac{1}{\tau_{T}}\left[g_{i}(\mathbf{x}, t)-g_{i}^{e q}(\mathbf{x}, t)\right]+\frac{1}{b_{T}} S,
$$

where $\tau_{T}$ is the relaxation time for heat conduction (diffusion) and $S$ is the heat source/sink. According to Toffoli and Margulos (1990), it seems sufficient to use a square or a cubic lattice in two or three dimensions respectively, to yield full isotropy for diffusive phenomena. The equilibrium functions for the D2Q4 velocity model are defined as follows:

$$
g_{i}^{e q}(\mathbf{x}, t)=\frac{1}{4} T\left[1+3\left(\mathbf{e}_{i} \cdot \mathbf{v}\right)\right] \quad \forall i=1, \ldots, 4,
$$

where $\boldsymbol{v}$ is defined from eq. (5) as $\mathbf{v}=\rho \mathbf{v} / \rho$ and the temperature $T$ is expressed as the following sum:

$$
T=\sum_{i=0}^{b_{T}} g_{i}
$$

Fig. 4 illustrates dependence of the temperature on inner energy, where the commonly used inverse function $Q=f(T)$ is applied. Line 1 represents a material with constant thermal properties and linear inner energy dependence. Such material is characterised by the constant value of $\rho c$, where $\rho$ is the density and $c$ is the specific heat. Line 2 represents a non-linear behaviour, typical for the phase transition, when solidus and liquidus temperatures are different. The three different line fragments are characterised by the different specific heat values $\rho c$ corresponding to solid, mixed and liquid phases. The "effective specific heat" method is used for characterisation of the middle fragment. The standard LBM can be applied for the mentioned two cases. However, it cannot be used for the case presented by line 3 because $\rho c$ arises to infinity in the middle horizontal part of the line (Fig. 4). The approach described in this section can be successfully applied both for this and for the first two cases. Obviously, the temperature dependence on inner energy can be arbitrary nonlinear, as it is expressed by line 4 in Fig. 4.

\section{LBM formulation of the thermal problem}

The energy balance equation can be presented in the following form: 


$$
\rho c \frac{\partial T}{\partial t}+\nabla(\rho c \mathbf{v} T)=\nabla(k \nabla T)+S
$$

where: $k$ is the thermal conductivity. Considering the one dimensional problem without advection and heat sources, the eq. (10) can be reduced to the heat conduction equation:

$$
\rho c \frac{\partial T}{\partial t}=\frac{\partial}{\partial x}\left(k \frac{\partial T}{\partial x}\right)=\frac{\partial k}{\partial x} \frac{\partial T}{\partial x}+k \frac{\partial^{2} T}{\partial x^{2}}
$$

The only second term remains in the right side of the eq. (11) when the thermal conductivity $k$ is a constant in the considered domain. Several points of the discrete domain are schematically presented in Fig. 5. The right side of eq. (11) can be presented in the following discrete form:

$$
k \frac{\partial^{2} T}{\partial x^{2}} \approx k \frac{T^{-}-2 T+T^{+}}{2 \delta x^{2}}
$$

The distribution functions $f_{i}$ and $f_{i}^{\text {eq }}$ can be considered as heat fluxes depending only on the temperature and not depending on the temperature gradient:

$$
f_{i}=k w_{i} T^{-(+)}, f_{i}^{\mathrm{eq}}=k w_{i} T
$$

The right side of eq. (11) can be considered as balance between the incoming and outcoming heat fluxes at the appropriate point. Their distribution along the main directions can be presented in the following form:

$$
\begin{aligned}
& k \frac{\partial^{2} T}{\partial x^{2}} \approx \frac{1}{2 \delta x^{2}} \sum \frac{f_{i}-f_{i}^{\mathrm{eq}}}{w_{\mathrm{i}}} \\
& \frac{\partial^{2} T_{i}}{\partial x^{2}} \approx \frac{f_{i}-f_{i}^{\mathrm{eq}}}{2 k w_{\mathrm{i}} \delta x^{2}}
\end{aligned}
$$

The left side of eq. (11) can be considered as a change of inner energy $Q$ of the material at the representative point (volume), $Q=\rho c T$, which can also be distributed along the main directions:

$$
\begin{aligned}
& \rho c \frac{\partial T}{\partial t}=\frac{\partial Q}{\partial t}=\sum \frac{\partial Q_{i}}{\partial t} \approx \sum \frac{Q_{i}(t+\delta t)-Q_{i}(t)}{\delta t} \\
& =\sum \frac{f_{i}(t+\delta t)-f_{i}(t)}{\delta t}
\end{aligned}
$$

Joining eq. (15) and eq. (16), the lattice Boltzmann equation is obtained as follows:

$$
f_{i}(t+\delta t)=f_{i}(t)-\frac{1}{\tau}\left[f_{i}(t)-f_{i}^{\mathrm{eq}}(t)\right] .
$$

The main difference of eq. (17) from commonly used eq. (2), or eq. (7), is the physical interpretation of the distribution function $f$. The distribution function is the distribution of inner energy rather than a temperature distribution. The inner energy $Q$ can be calculated directly from the following distribution function: 


$$
Q=\sum f_{i}
$$

The replacment of the temperature $T$ by the inner energy $Q$ allows for obtaining an easy solution of the melting-solidification problem (line 2 in Fig. 4), especially when solidus and liquidus are the same temperatures (line 3 in Fig. 4). However, it should be noted that such replacement makes calculation algorithm somewhat more complicated and it is not relevant for homogeneous materials, such as those expressed by line 1 in Fig 4. The solution algorithm for the collision stage is the following:

1. The new distribution functions $f_{i}$ are calculated according to eq. (17) based on current values;

2. The inner energy $Q$ is calculated according to eq. (18);

3. The temperature $T$ is calculated on the basis of its actual dependence on inner energy $Q$ (Fig. 4): $T=f(Q), \Delta T=\Delta Q / \rho c$ for homogenous medium;

4. The new distribution functions are calculated according to eq. (13) during the streaming stage.

To illustrated the developed approach, the results of 1D simulation of the thermal problem are presented in Fig. 6. The liquid with freezing point at $0^{\circ} \mathrm{C}$ and initial temeperature of $20^{\circ} \mathrm{C}$ cools down maintaining the left side of the $1 \mathrm{D}$ domain at the constant temperature of $-20^{\circ} \mathrm{C}$. In this particular case, the liquidus and solidus temperatures are the same. The thermal parameters of the material in liquid and solid state are different. The model also takes into account the heat of transformation. Fig. 6 illustrates the temperature distribution along the 1D domain predicted for the consequent stages of cooling including freezing.

\section{Mechanical solid-liquid interface - material flow}

The molten material flow is simulated within the frame of the free surface LBM model with consideration of gravity, varying surface tension, viscosity and wettability. The latest state-of-the-art numerical methods applied for simulation of the processes in the gas(liquid)-particle systems (with full 3D description of the geometry) are the direct numerical simulation methods coupled with discrete element methods (Deen et al., 2014) and immersed boundary method (IBM) (Tavassoli Estahbanati et al., 2013), computational fluid dynamics (CFD) (Perig and Golodenko, 2014) or LBM (Rong et al., 2014 and Grucelski and Pozorski, 2013). LBM allows for flow calculations within any geometry (large or small scale) without generation of complex numerical meshes. The recently developed LBM based models show sufficiently high stability for modelling both laminar and turbulent flows in complex geometries (Geier, 2006, Straka, 2016 and Mohamad, 2011). Fig. 7 and 8 illustrate some examples of 2D and 3D solutions obtained by the authors using LBM based models demonstrating high ability of LBM for modelling of fluid flows coupled with heat transfer.

\section{Conclusions}

A new holistic model has been developed and presented in the paper. Proposed homogenous model based on combination of cellular automata and Lattice Boltzmann methods significantly simplifies interface and 
interaction between different parts of the holistic model in comparison with other commonly used multi-scale approaches. The laser assisted ALM process is analised and divided on several stages considering the relevant physical phenomema. The entire holistic model consists of four interrelated submodels currently developed to a different extent.

1. The CA based model of powder bed generation is in the final stage of developemnt. It is being extended to application of irregular particle shape and size distributions and also optimization of its interface with LBM models.

2. The LBM-CA based model of heat exchange and transfer is being developed, currently allowing for 2D simulations. It is going to be followed by its $3 \mathrm{D}$ extension and adaptation to the holistic model.

3. The thermal solid-liquid interface model is at an early stage of development (1D version). It is expected to be extended to 2D and 3D domain.

4. The mechanical solid-liquid interface model has been developed for continuous liquid flow. Consideration in the model and including of free surface flow, wettability, surface tension and other relevant phenomena will now have to take the matter further.

\section{Acknowledgments}

The support of the National Science Centre Poland (grant no. DEC-2013/09/B/ST8/00141) and EPSRC UK under grant EP/L505158/1 is greatly appreciated.

\section{References}

Deen, N. G., Peters, E. A. J. F., Padding, J. T. and Kuipers, J. A. M., (2014), "Review of direct numerical simulation of fluid-particle mass, momentum and heat transfer in dense gas-solid flows", Chem. Eng. Sci., vol. 116, pp. 710-724.

Domingos, M., Chiellini, F., Gloria, A., Ambrosio, L., Bartolo, P., and Chiellini, E., (2012), "Effect of process parameters on the morphological and mechanical properties of 3D Bioextruded poly(E-caprolactone) scaffolds", Rapid Prototyping J., vol. 18, no. 1, pp. 56 - 67.

Eshghinejadfard, A. and Thévenin, D., (2016), "Numerical simulation of heat transfer in particulate flows using a thermal immersed boundary lattice Boltzmann method", Int. J. Num. Meth. Heat \& Fluid Flow, Vol. 60, pp. 31-46.

Eshraghi, S. and Das, S. (2012), "Micromechanical finite-element modelling and experimental characterisation of the compressive mechanical properties of polycaprolactone-hydroxyapatite 
composite scaffolds prepared by selective laser sintering for bone tissue engineering", Acta Biomaterialia, vol. 8, pp. 3138-3143.

Geier, M.C., (2006), “Ab Initio Derivation Of The Cascaded Lattice Boltzmann Automaton”, Ph.D. thesis, University of Freiburg - IMTEK.

Grucelski, A., and Pozorski, J., (2013), "Lattice Boltzmann simulation of flow past an obstacle and in simple porous media", Comp. Fluids, vol. 71, pp. 406-416.

Hench, L. L., and Polak J. M., (2002), “Third-generation biomedical materials”, Science, vol. 295, no. 5557, pp. 1014-1017.

Kremer, G. M., (2010), “An Introduction to the Boltzmann Equation and Transport Processes in Gases", Springer.

Krzyzanowski, M., Svyetlichnyy, D., Stevenson, G., and Rainforth, W. M., (2016), "Powder bed generation in integrated modelling of additive layer manufacturing of orthopaedic implants", Int. J. Adv. Manuf. Technol. doi: 10.1007/s00170-016-8491-x.

Kuwata, Y. and Suga, K., (2016), "Lattice Boltzmann direct numerical simulation of interface turbulence over porouse and rough walls", Int. J. Num. Meth. Heat \& Fluid Flow, doi: 10.1016/j.ijheatfluidflow.2016.03.006.

Lam, C. X. F., Teoh, S. H., and Hutmacher, D. W., (2007), "Comparison of the degradation of polycaprolactone and polycaprolactone-( $\beta$-tricalcium phosphate) scaffolds in alkaline medium", Polymer Int., vol. 56, no. 6, pp. 718-728.

Mercuri, L.G., Wolford, L.M., Sanders, B., White, D., Hurder, A., and Herderson, W., (1995), "Custom CAD/CAM total temporomandibular joint reconstruction system: preliminary multicenter report", J. Oral Maxillofacial Surg., vol. 53, pp. 106-115.

Mikhaylovska, T. V., (2008), "Mathematical modeling of metallurgical processes with phase transformation on base of cellular automata", PhD thesis, National Metallurgical Academy of Ukraine.

Mohamad, A.A., (2011), "Lattice Boltzmann Method. Fundamentals and Engineering Applications with Computer Codes", Springer-Verlag London.

Perig, A. V., and Golodenko, N. N., (2014), "CFD 2D simulation of viscous flow during ECAE through a rectangular die with parallel slants”, Int. J. Adv. Manufac. Technol., vol. 74, no. 5-8, pp. 943-962.

Rong, L. W., Dong, K. J., and Yu, A. B., (2014), "Lattice-Boltzmann simulation of fluid flow through packed beds of spheres: Effect of particle size distribution”, Chem. Eng. Sci., vol. 116, pp. 508-523. 
Straka, R., (2016), "Numerical simulation of heat transfer in packed beds by two population thermal Lattice Boltzmann method", Mechanics \& Industry, vol. 17, no.2, art. no. 203, pp.1-11.

Svyetlichnyya, D. S., (2014), "A three-dimensional frontal cellular automataon model for simulation of microstructure evolution — initial microstructure module", Modeling Simul. Mater. Sci. Eng., vol. 22, no. 085001.

Svyetlichnyy, D. S., (2013), “Frontalne automaty komórkowe do modelowania mikrostruktury”, Wydawnictwa AGH.

Svyetlichnyyb, D. S., (2014), "Three-dimensional frontal cellular automata model of microstructure evolution phase transformation module”, ISIJ Int., vol. 54, pp. 1386-1395.

Svyetlichnyy, D. S., (2010), "Modeling of microstructure evolution in process with severe plastic deformation by cellular automata", Mater. Sci. Forum, vol. 638-642, pp. 2772-2777.

Svyetlichnyy, D., Majta, J., and Muszka, K., (2008), "Modeling of microstructure evolution of BCC metals subjected to severe plastic deformation”, Steel Res. Int., vol. 79, pp. 452-458.

Svyetlichnyy, D. S, Muszka, K, and Majta, J., (2015), “Three-dimensional frontal cellular automata modeling of the grain refinement during severe plastic deformation of microalloyed steel", Comput. Mater. Sci., vol. 102, pp. 159-166.

Tammas-Williams, S., Zhao, H., Léonard, F., Derguti, F., Todd, I., and Prangnell, P. B., (2015) “XCT analysis of the influence of melt strategies on defect population in $\mathrm{Ti}-6 \mathrm{Al}-4 \mathrm{~V}$ components manufactured by Selective Electron Beam Melting", Mater. Characterization, vol. 102, pp. 47-61.

Tavassoli Estahbanati, H., Kriebitzsch, S. H. L., Hoef, M. A., van der, Peters, E. A. J. F., and Kuipers, J. A. M., (2013) "Direct numerical simulation of particulate flow with heat transfer", Int. J. Multiphase Flow, vol. 57, pp. 29-37.

Toffoli, T., and Margolus, N., (1990), “Invertible cellular automata: A review”, Physica D, vol. 45, pp. 229253.

Williams J. M., Adewunmi, A., Schek, R. M., Flanagan, C. L., Krebsbach, P. H., Feinberg, S. E., Hollister, S. J., and Das, S., (2005), "Bone tissue engineering using polycaprolactone scaffolds fabricated via selective laser sintering", Biomaterials, vol. 26, no. 23, pp. 4817-4827.

Wu, G., Zhou, B, Bi, Y., and Zhao, Y., (2008), "Selective laser sintering technology for customized fabrication of facial prostheses", J Prosthet Dent., vol. 100, no. 1, pp. 56-60.

Zarghami, A., Ubertini, S. and Succi, S., (2014) "Finite volume formulation of thermal lattice Boltzmann method, Int. J. Num. Meth. Heat \& Fluid Flow, Vol. 24 No. 2, pp. 270-289. 



\section{Figure captions}

Fig. 1 Selective laser sintering/melting (SLS/SLM).

Fig. 2 Holistic model for simulation of SLS/SLM process.

Fig. 3 Simulated deposition of the irregular shape particles into a cylinder.

Fig. 4 Dependence of the temperature $T$ on inner energy $Q$ : different cases.

Fig. 5 Applied calculation scheme.

Fig. 6 Predicted temperature distribution illustrating cooling and freezing of the liquid.

Fig. 7 Velocity (a) and temperature (b) distribution during 2D fluid flow predicted using LBM.

Fig. 8 Velocity distribution during 3D fluid flow predicted using LBM. 


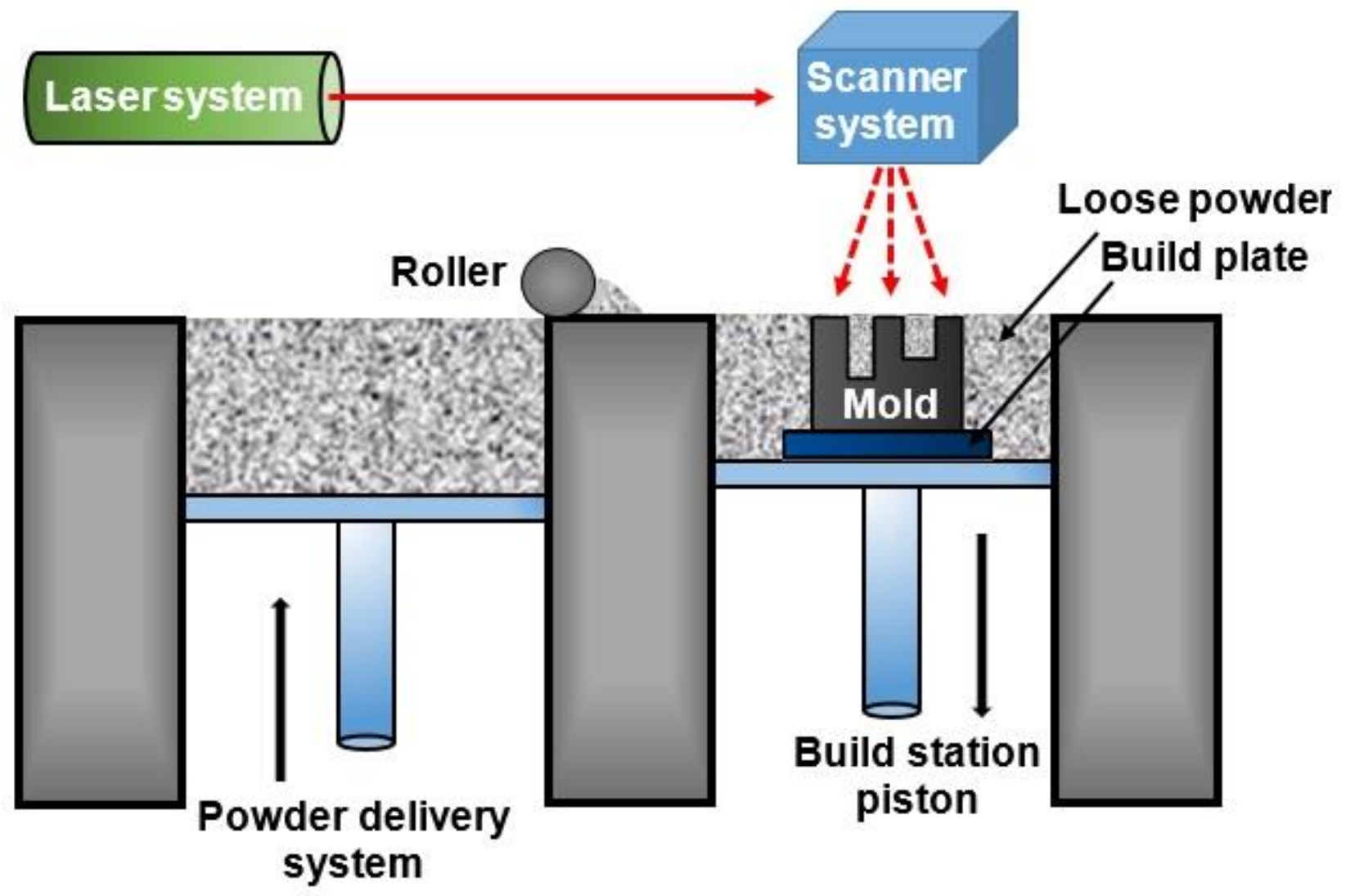

Fig. 1 Selective laser sintering/melting (SLS/SLM) 


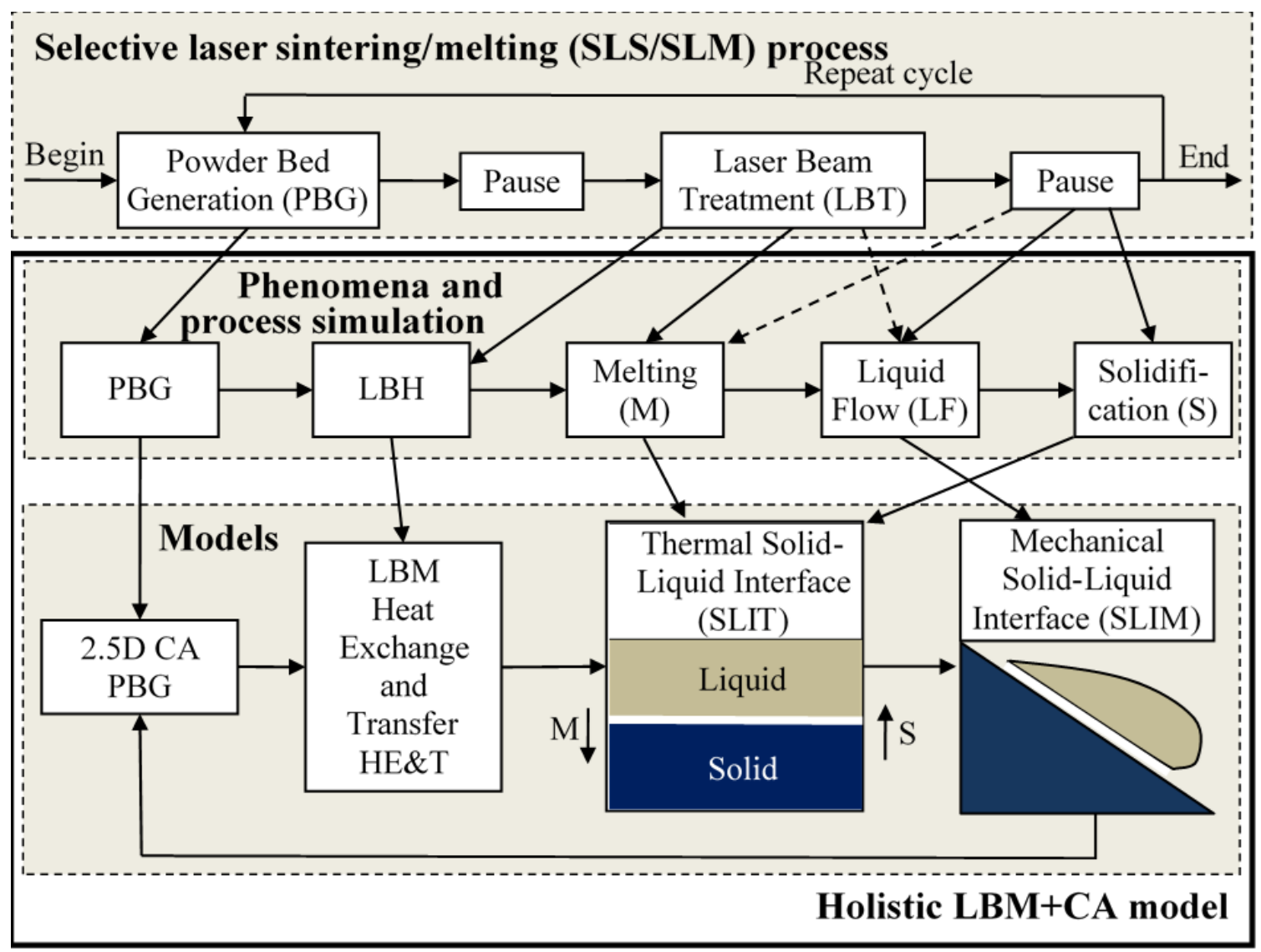

Fig. 2 Holistic model for simulation of SLS/SLM process. 


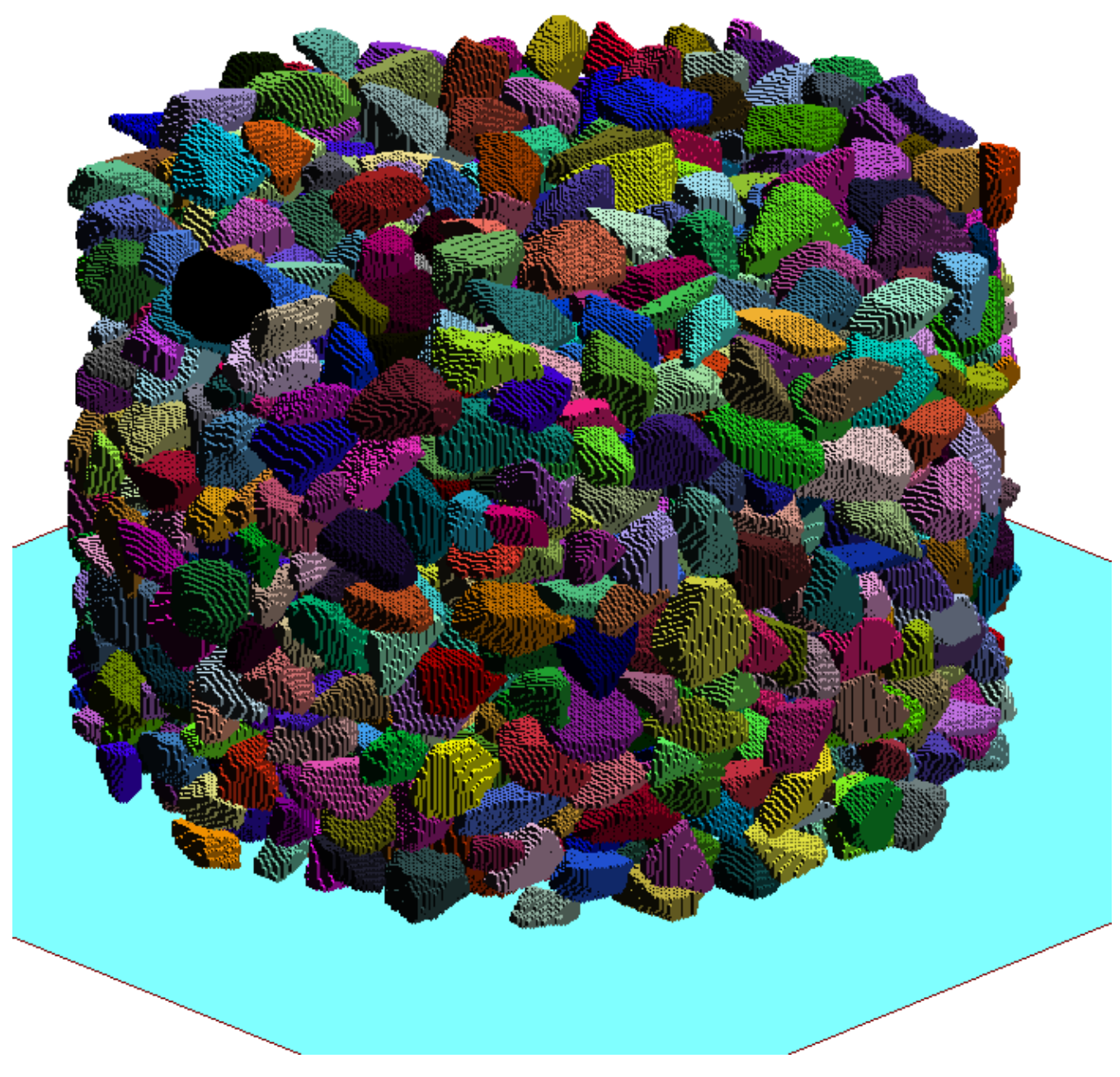

Fig. 3 Simulated deposition of the irregular shape particles into a cylinder. 


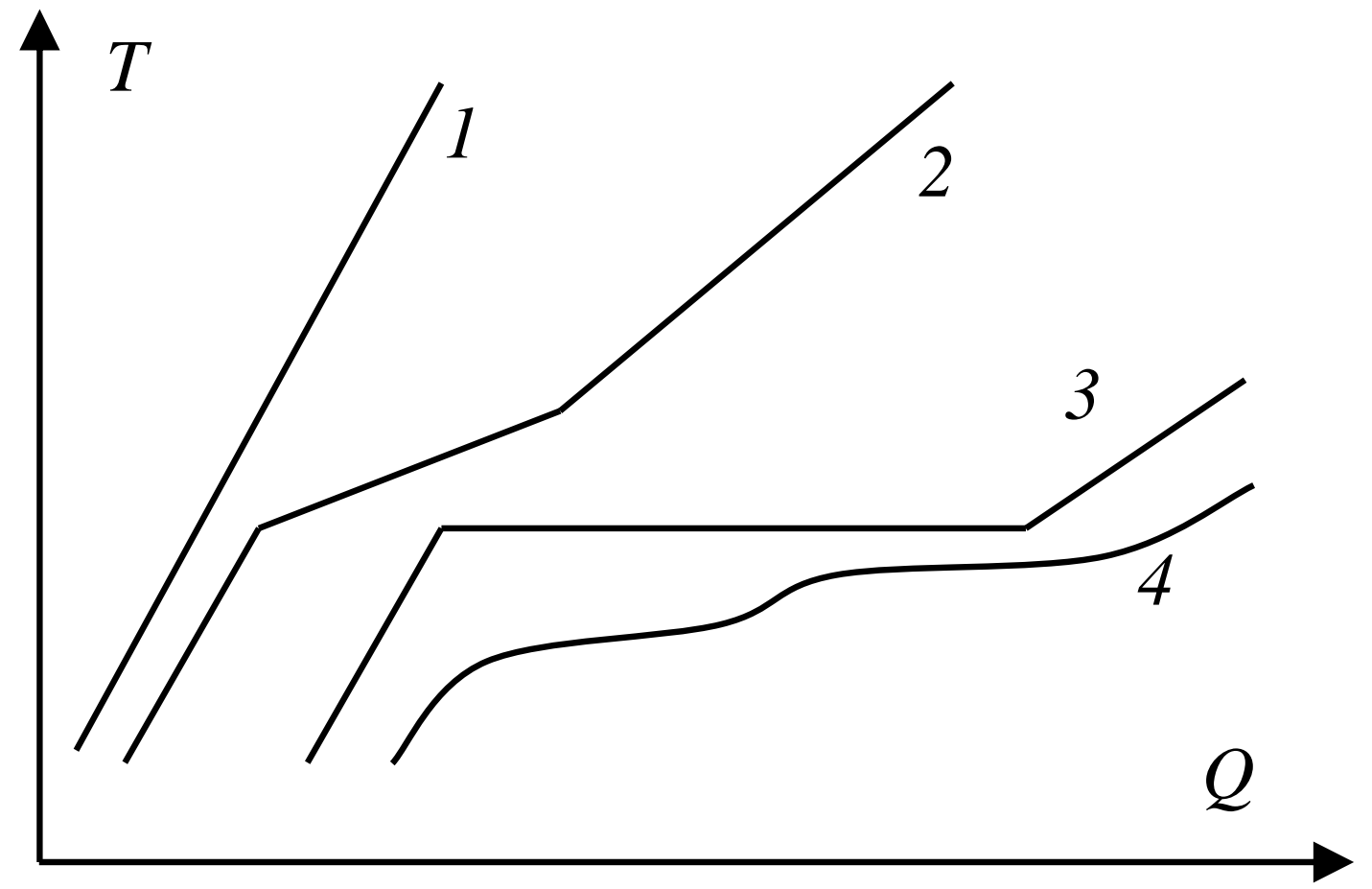

Fig. 4 Dependence of the temperature $T$ on inner energy $Q$ : different cases. 


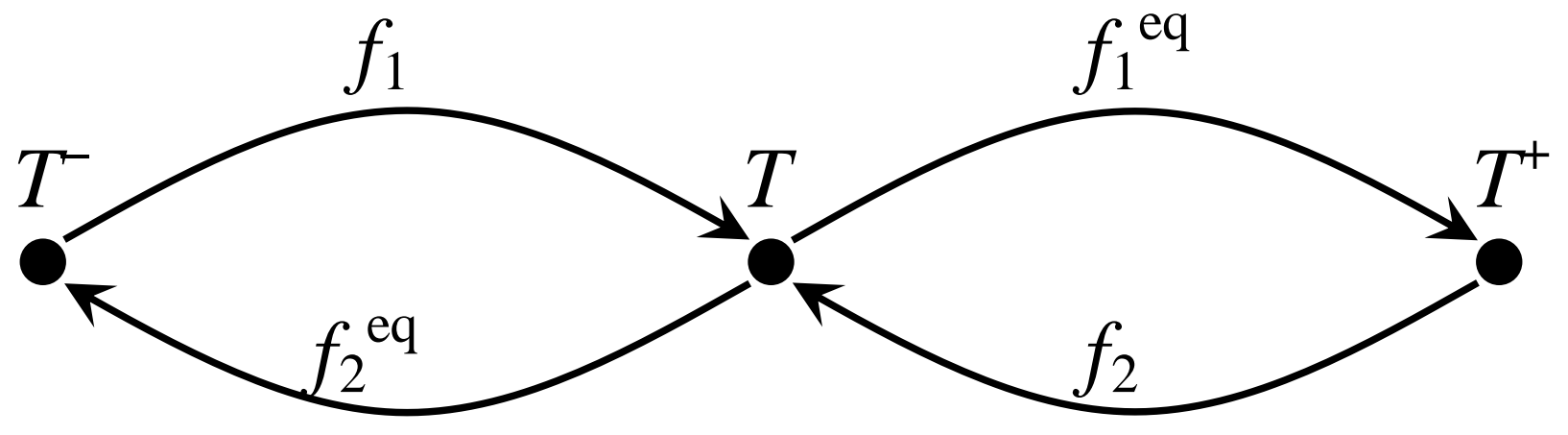

Fig. 5 Applied calculation scheme. 


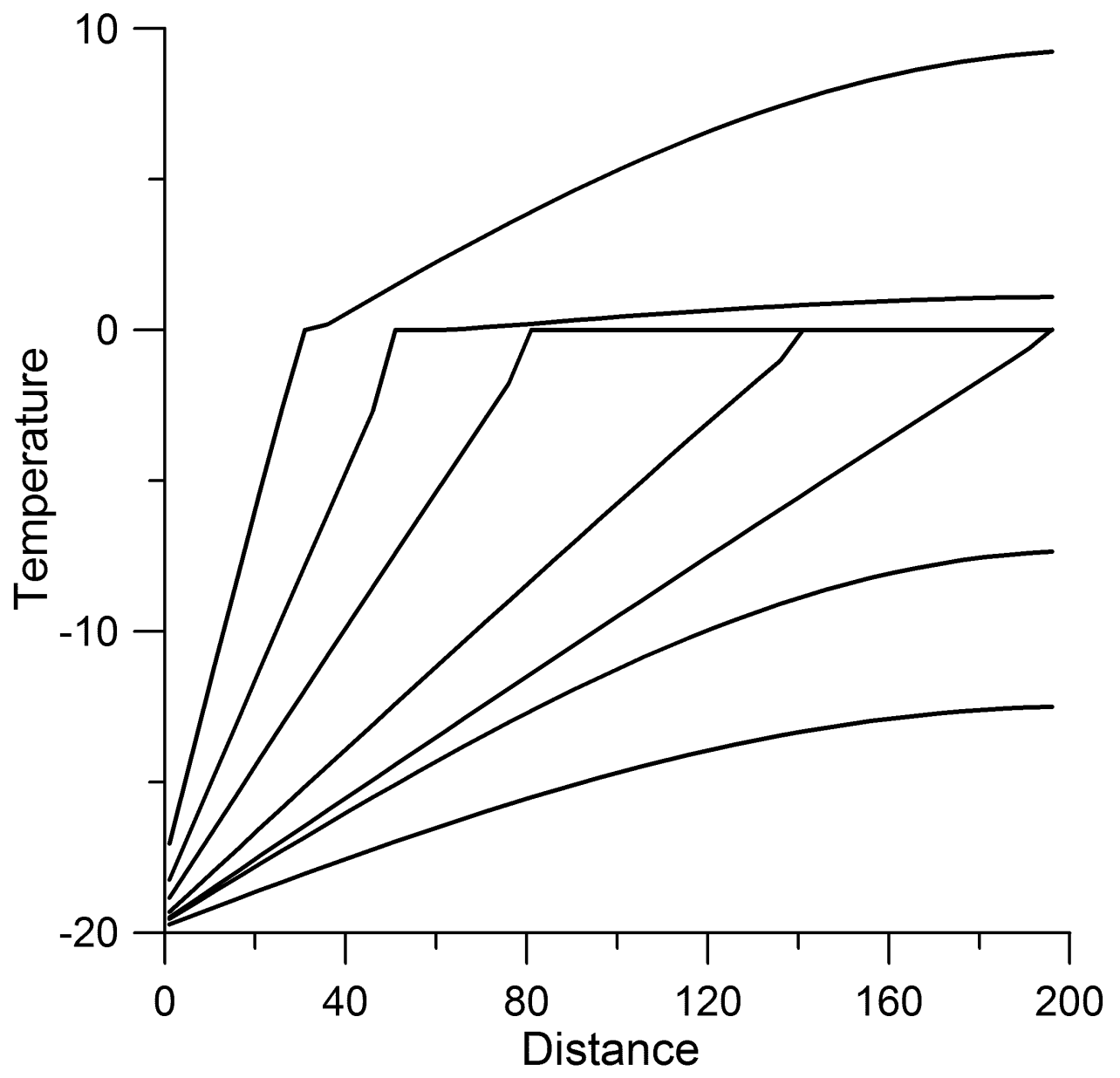

Fig. 6 Predicted temperature distribution illustrating cooling and freezing of the liquid. 

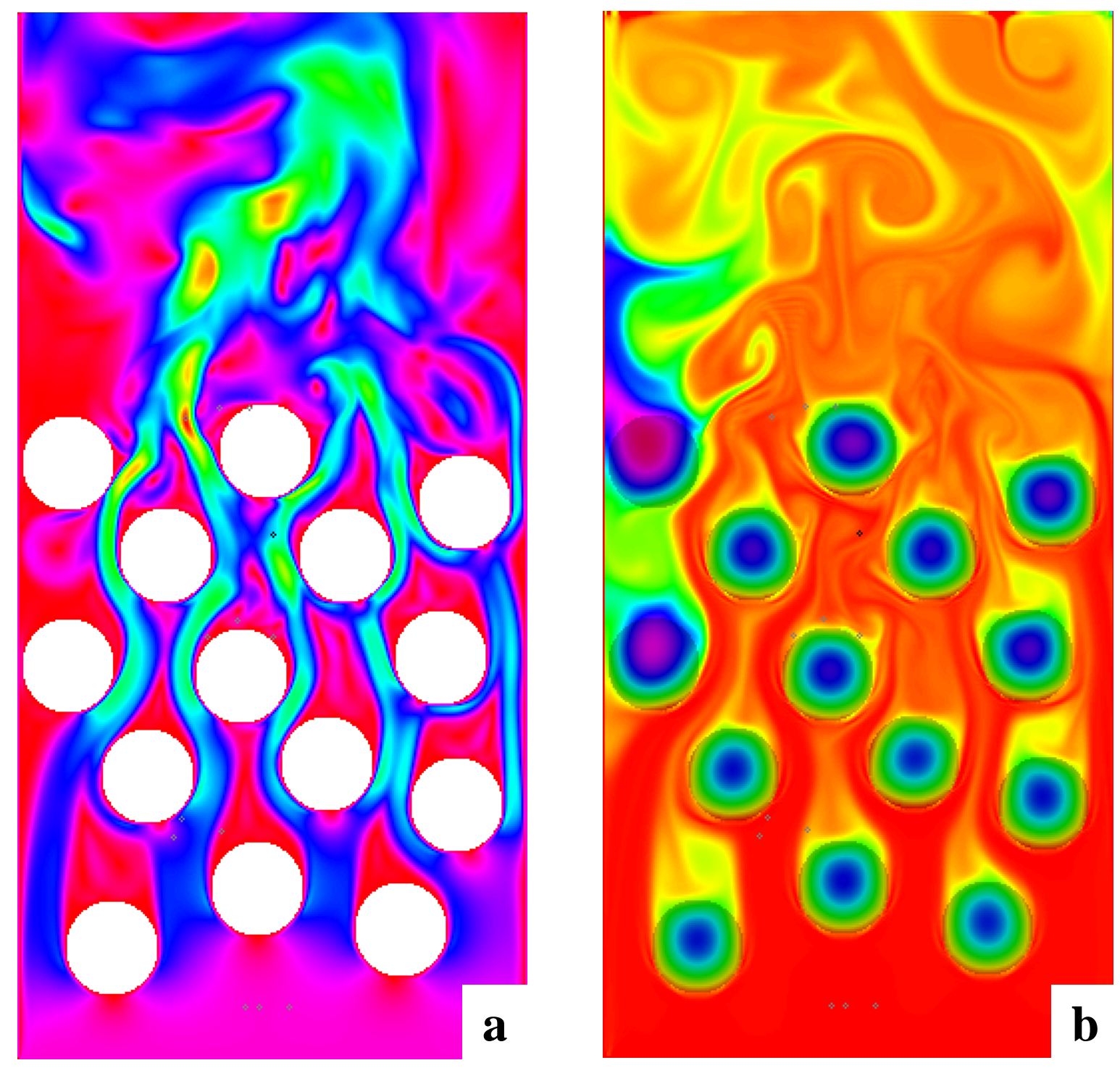

Fig. 7 Velocity (a) and temperature (b) distribution during 2D fluid flow predicted using LBM. 


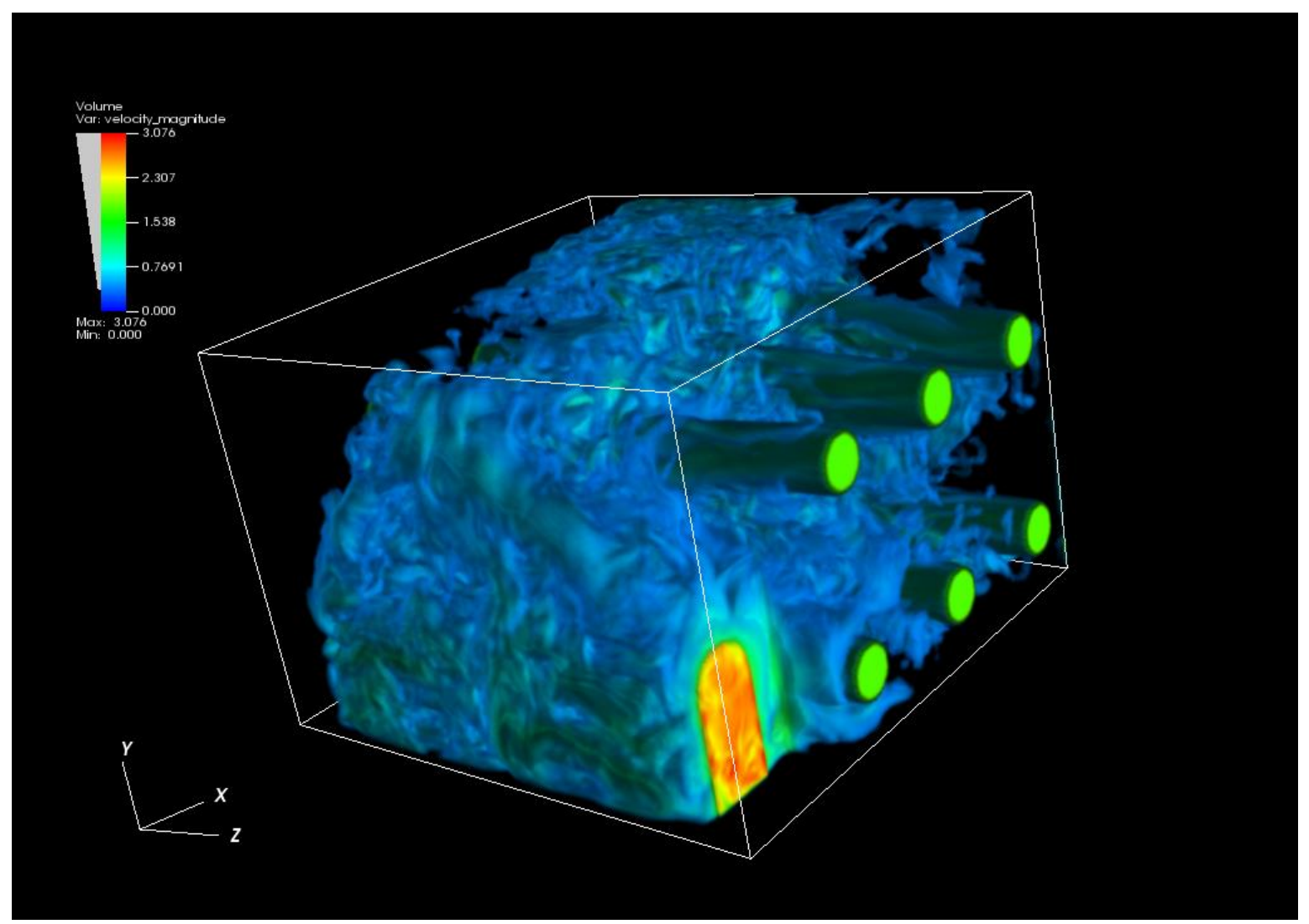

Fig. 8 Velocity distribution during 3D fluid flow predicted using LBM. 